\title{
Postoperative ileus: in search of an international consensus on definition, diagnosis, and treatment
}

\author{
Daniel Gero $^{1}$ - Olivier Gié ${ }^{1}$ - Martin Hübner ${ }^{1} \cdot$ Nicolas Demartines $^{1} \cdot$ Dieter Hahnloser $^{1}$
}

Received: 21 May 2016 / Accepted: 17 July 2016/Published online: 3 August 2016

(C) Springer-Verlag Berlin Heidelberg 2016

\begin{abstract}
Purpose Postoperative ileus (POI) is a frequent complication after abdominal surgery; nonetheless, it remains poorly defined. Our aim was to achieve an international consensus among leading colorectal surgeons on definition, prevention, and treatment of POI.

Methods Thirty-five experts from five continents participated in a three-round Delphi process. Round 1 contained openended questions on POI and postoperative nausea and vomiting (PONV). Round 2 included closed-ended questions. Round 3 measured agreement on a 5-point Likert scale. Consensus was defined when items were rated as agree or strongly agree by at least $70 \%$ of the experts.

Results Experts reached following consensus: POI is a temporary inhibition $(86 \%)$ of gastrointestinal motility after surgical intervention due to non-mechanical causes $(89 \%)$ and prevents sufficient oral intake ( $96 \%$ ). Abdominal distension/ tenderness are the most relevant clinical signs $(71 \%)$. Nasogastric tube placement is not mandatory (78 \%) but can be removed without previous clamping $(81 \%)$ /gastrointestinal contrast study $(100 \%)$. Preventive measures are recommended to decrease the risk of POI (96\%): narcotic sparing analgesia $(89 \%)$ and fluid optimization (74\%). Treatment of
\end{abstract}

Presented as oral communication: 102th Annual Congress of the Swiss Society of Surgery, 20-22 May 2015, Bern, Switzerland

Electronic supplementary material The online version of this article (doi:10.1007/s00423-016-1485-1) contains supplementary material, which is available to authorized users.

Dieter Hahnloser

dieter.hahnloser@chuv.ch

1 Department of Visceral Surgery, University Hospital CHUV, Rue du Bugnon 46, CH-1011 Lausanne, Switzerland
POI should include stimulation of ambulation (96\%) and stop of opioids $(74 \%)$. Total parenteral nutrition is recommended from the 7th day without sufficient oral intake ( $81 \%)$. There was no consensus on the ranking of POI's symptoms, on the imaging modality of choice for the diagnosis of POI, neither on the difference between POI and PONV.

Conclusions This Delphi study achieved consensus on the definition, relevant clinical signs, prevention, treatment, and supportive care of POI. Areas of non-consensus were identified (necessity and modality of radiologic imaging to establish the diagnosis, difference between POI and PONV), giving opportunity for further research.

Keywords Postoperative ileus · Ileus · Delphi method · PONV $\cdot$ Postoperative nausea and vomiting $\cdot$ Consensus

\section{Introduction}

The definition of postoperative ileus (POI) is heterogeneous and for this reason, its reported incidence, diagnostic algorithm, prevention and therapeutic measures underlie wide variation [1]. POI is often described as a pathologic pattern of gastrointestinal (GI) motility occuring after any type of major surgery, with increased length of stay [2]. Typical signs and symptoms are inability to tolerate oral diet, nausea, vomiting, absence of effective bowel movements, abdominal distention, and discomfort [3]. As a consequence, POI temporarily alters the quality of life and imposes a great economic burden to the health-care systems, with an estimated annual cost in the USA between $\$ 0.75$ and 1.5 billion $[4,5]$.

Due to its high prevalence and debilitating effect, and in the era of enhanced recovery, studies on etiology, risk factors, and treatment of POI are in the focus of medical literature. On the other hand, distinction between postoperative nausea and 
vomiting (PONV), a physiologic period of gastrointestinal dismotility following abdominal surgery and POI varies arbitrarily among studies [6-8]. Consensual distinction or merging of those clinical entities according to precise criteria, such as their occurrence in the postoperative course or the hierarchy of their symptoms seems to be necessary. Homogenization of the definitions of these frequent postoperative complications would help to identify primary end-points for future prospective studies targeting the optimization of the management of patients presenting POI.

The aim of the present study was to achieve an international consensus among leading colorectal surgeons on the definition, prevention, and treatment of postoperative ileus using the Delphi method.

\section{Material and methods}

A three-round Web-based Delphi approach was used in this consensus process. The Delphi technique is an iterative process used to collect and distill the judgments of a group of experts to reach consensus on specific questions or issues [9]. Fiore et al. used the technique to define discharge criteria after colorectal surgery - their paper served as model for the design of the current study [10]. Delphi research involves completion of structured questionnaires interspersed with summary and feedback derived from previous responses [11]. Experts remain anonymous to each other during the process. Advantages of the Delphi over usual consensus methods are the (a) non-necessity of face-to-face meetings enabling the sharing of personal views without embarrassment and (b) the possibility to simultaneously survey experts from all around the world to find a consensus $[12,13]$. The original Delphi method was developed in the 1950s for a US sponsored military project, and since, it has been widely used for similar studies in health care $[14,15]$ and surgery $[16,17]$.

\section{Expert nomination and recruitment}

Surgical program directors, chairmen of colorectal surgery departments, and academic surgeons with publications involving POI were identified using the 2013 Yearbook of the Fellow of the American Society of Colon and Rectal Surgeons, the European Society of Colorectal Surgeon member list and the Pubmed database. They were deemed as "experts."

To ensure the international standpoint to this consensus, a panel of 35 experts with 10 experts from North and South America, 10 from Asia/Oceania, and 15 from Europe was targeted. A recruitment letter in English was sent via e-mail providing a brief outline of the project and its objectives. If invited experts did not respond to the invitation within 4 weeks, a reminder was sent out. Based on the same selection criteria, further experts were invited if no answer came in the next 4 weeks or the expert declined to participate in the study. The recruitment process was continued until a total of 35 experts from the 3 geographic areas agreed to participate in the study. A positive response to the recruitment letter served as informed consent.

\section{Delphi process}

In all 3 rounds, questionnaires were sent by e-mail via secured web-link. A password-protected, Web-based interface was used for creating, publishing and analyzing surveys (SurveyMonkey $\odot$, Palo Alto, CA, USA). Before the start of the first round, the questionnaire was pilot tested among all $(n=33)$ physicians of the Department of Visceral Surgery of the University of Lausanne. Minor changes in structure and word use were made in response to their feedback. Experts were given 3 weeks to respond to each round. A reminder to complete the questionnaire was sent at 4 weeks and repeated three times by 3 weeks intervals. An interval of 6 weeks between rounds was used to summarize the data and develop the next questionnaire.

The round 1 questionnaire included ten open-ended questions (Table 1). Responses obtained in round 1 were summarized in a Microsoft Excel table. A qualitative thematic analysis was jointly performed by all authors of the study; similar patterns in the answers were simplified to keywords and grouped in separate columns. The round 2 consisted of closed-ended questions, such as yes/no questions, multiple choice questions, or ranking. Questions were grouped in sets, corresponding to each of the open-ended questions of round 1 . Each question challenged the acceptance of an item/items mentioned by at least two experts in round 1 . Room for comments was provided at the end of each set.

In the third and final round, structured results and comments obtained to round 2 questions was provided and experts were asked to rank their agreement on each item by the use of a 5-point Likert scale (1, strongly disagree; 2, disagree; 3, neither agree nor disagree; 4 , agree; 5 , strongly agree). They could change their own round 2 ranks in light of their colleagues' responses and arguments. Room for comments was again provided at the end of each set.

\section{Data analyses}

Data were analyzed by using descriptive statistics. Mean ranks, standard deviation, and percentage agreement obtained for each question was calculated with the use of the SurveyMonkey online interface and Microsoft Excel. Consensus was defined when at least $70 \%$ of the experts in round 3 agreed or disagreed (i.e., they rated either 4 or 5 or 1 or 2 on the Likert scale, respectively) [18]. In round 3, the means of mean Likert scores per item per geographic region 
Table 1 Round 1 questions and respective answers mentioned by at least two experts
How would you define postoperative ileus (POI)?

Which elements of the clinical examination do you find relevant for the diagnosis of POI?

Which features of the gastrointestinal transit would you consider relevant for the diagnosis of POI?

Which radiologic imaging technique do you use to establish the diagnosis of POI?

How would you specify postoperative nausea and vomiting (PONV)?

How do you differentiate PONV and POI?

What are your indications for the placement of a nasogastric (NG) tube in patients with POI?

On which criteria do you rely to remove the nasogatric tube in patients with POI?

Do your patients receive prophylactic medication to prevent POI? Which are those?

How do you treat POI? (Please explain in detail)
Lack of effective bowel movements, reference on timing, nausea/vomiting, no mechanical obstruction, necessity of an NG tube, prevents oral intake

Abdominal distension, nausea/vomiting, pain, auscultation

Absence of gas/stool, impaired food tolerance, nausea/ vomiting, delay in normal bowel function

No radiologic exam, abdominal X-ray, abdominal Xray $+\mathrm{CT}$ if needed, abdominal CT alone, water-soluble contrast agent

Nausea/vomiting, medication (anesthesia) induced, occurs in the first $24 \mathrm{~h}-48 \mathrm{~h}$ postoperatively

Abdominal distension in POI, duration of PONV: 1 day, no bowel function impairment in PONV

Vomiting, vomiting more than twice, abdominal distension/discomfort

Gas/stool, amount/24 h less than 1000/500 ml, no abdominal distension, clamping test

No, metoclopramid, ondasentron, dexamethasone, narcotic sparing analgesia, alvimopan

NG tube, wait and see, total parenteral nutrition, stop opioids, metoclopramid, neostigmine, mobilization, ondasentron

POI postoperative ileus, $N G$ nasogastric, $C T$ computed tomography, $P O N V$ postoperative nausea and vomiting

were compared by the Chi-square goodness-of-fit statistical test for independence.

\section{Results}

\section{Expert recruitment}

A total of 61 experts were initially invited, and finally, 35 experts (10 from the Americas, 10 from Asia/Oceania, and 15 from Europe) did agree to participate in the study (Fig. 1). Due to the high rate of non-responders and refusals, recruitment of the experts took more time than initially planned (4 months versus 1 month). All experts were able to communicate effectively in English. The rate of included experts completing each round was $69 \%(n=24), 80 \%(n=28)$, and $80 \%(n=28)$ in the 1 st, 2 nd, and 3rd rounds, respectively.

\section{Round 1}

The thematic analysis of the responses obtained in round 1 identified items that were mentioned by at least two experts per question and are listed in Table 1.

\section{Round 2}

The round 2 questionnaire was based on the answers obtained in round 1 . The answers are presented in Online resource 1 and were used to create the round 3 questionnaire. Most relevant comments were: POI is a transient inhibition of GI motility in response to surgical trauma and resolves without surgical intervention; bowel sounds are irrelevant in the diagnosis of POI; POI is a subset of PONV; PONV is part of POI;

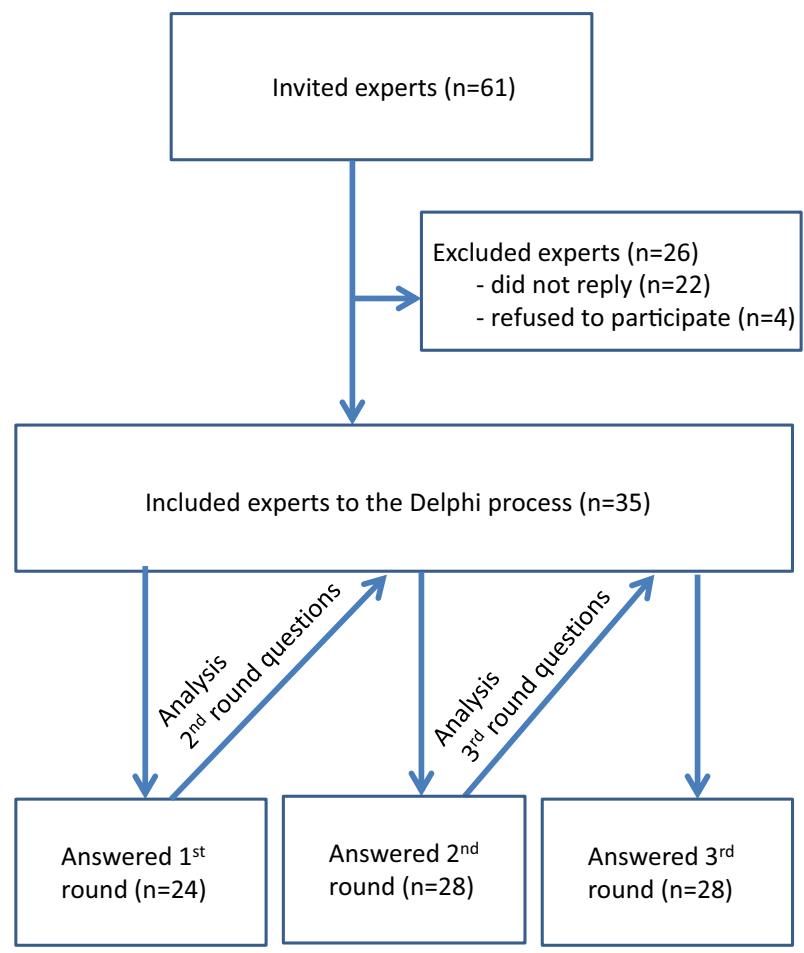

Fig. 1 Consort flowchart of experts' recruitment and participation 
exacerbating factors for PONV are opioid analgesics, intraperitoneal surgery, degree of bowel manipulation, and hypokalemia; appearance of bowel sounds and feeling of hunger pains is an indication of nasogastric (NG) tube removal.

\section{Round 3}

The questions and answers are presented in Fig. 2 and Online resource 2. Consensus was obtained in 20 items from a total of $57(35 \%)$. The definition, diagnosis, and treatment of POI in light of the results of this consensus research are listed in Table 2. Most relevant comments were: an expected temporary ileus is physiologic after surgery; flatus may be present with small bowel ileus; POI might recover earlier than the passage of gas/stool; POI is primarily a clinical diagnosis, investigations are indicated only if some complication is suspected; PONV is immediate and within hours of surgery; PONV is exacerbated by motion and frequently coincides with narcotic dose; NG tube is indicated for physical exam of gastric distention, abdominal distention, intractable nausea, vomiting one to two times; the use of laxatives is accepted but has never been studied; and antiemetic drugs do not treat POI. The means \pm standard deviations of the mean Likert scores per item per geographic region were $3.09 \pm 0.88$ for America, $3.18 \pm 0.66$ for Asia/Oceania, and $3.28 \pm 0.68$ for Europe. There was no significant difference between mean rankings per item between America and Asia/Oceania $(p=0.6)$ and America and Europe $(p=0.11)$; however, differences in experts' rankings between Asia/Oceania and Europe was at the limit of statistical significance $(p=0.049)$.

\section{a}

POI is present if 2 of 5 symptoms exist: 1.Nausea 2. Vomiting 3 . Inability to tolerate an oral diet over last $24 \mathrm{~h} 4$. Absence of flatus $\mathrm{POI}$ is defined as a transient inhibition of GI motility in response to surgical trauma and resolves without surgical intervention.

POl occurs from the 5th day after a surgical interventio

POI occurs from the 3rd day after a surgical intervention

POI occurs temporarily after a surgical interventio The definition of POI must include a reference on the timing of
POI.

The definition of $\mathrm{POI}$ must include that $\mathrm{POI}$ is due to nonmechanical causes

The definition of $\mathrm{POI}$ must include that $\mathrm{POI}$ prevents sufficient oral intake. The definition of POI must include that nausea and/or vomiting
are mandatory symptoms of POI.

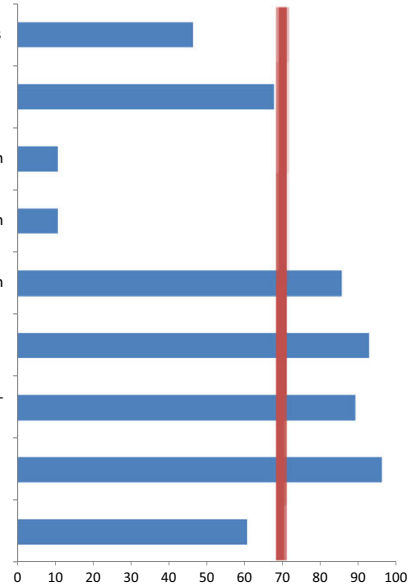

Fig. 2 Percentage agreement/disagreement in round 3 (red line, threshold of consensus). a Definition of postoperative ileus. b Diagnosis of postoperative ileus. c Difference between postoperative

\section{Discussion}

This Delphi study has provided partial consensus on the definition, prevention, and treatment of POI. The expert panel of leading colorectal surgeons from 5 continents, representing 23 countries agreed on the definition of POI, on its most relevant clinical signs and on key steps of preventive and therapeutic measures. Geographic localization did not seem to impede the consensus building process. Lack of consensus remained on the necessity and on the modality of radiologic imaging for the diagnosis of POI and on the difference between POI and PONV. The panel was rather well engaged in the Delphi process, reflected by the high response rates. In light of these factors, the consensual items gained from the Delphi process (Table 2) bear validity within the international academic surgical community.

\section{Definition of POI}

Experts agreed that POI prevents oral intake, that it occurs temporarily after a surgical intervention, and is due to nonmechanical causes. The most relevant clinical signs were abdominal distension and tenderness, and in third position, absence of normal bowel sounds. Experts did not differentiate $P O I$ and prolonged POI.

A systematic review on the definition of POI including 52 trials, found that the most frequently used criteria to establish the diagnosis of POI were the passage of flatus $(83 \%)$ and stool (79\%), ability to tolerate an oral diet (28\%), and presence of bowel sounds (13\%) [2]. Based on these findings and on a global survey, Vather et al. suggest the terminology "postoperative ileus" until the 3rd PO day when passage of flatus/stool and tolerance of an oral diet is not experienced.

\section{b}

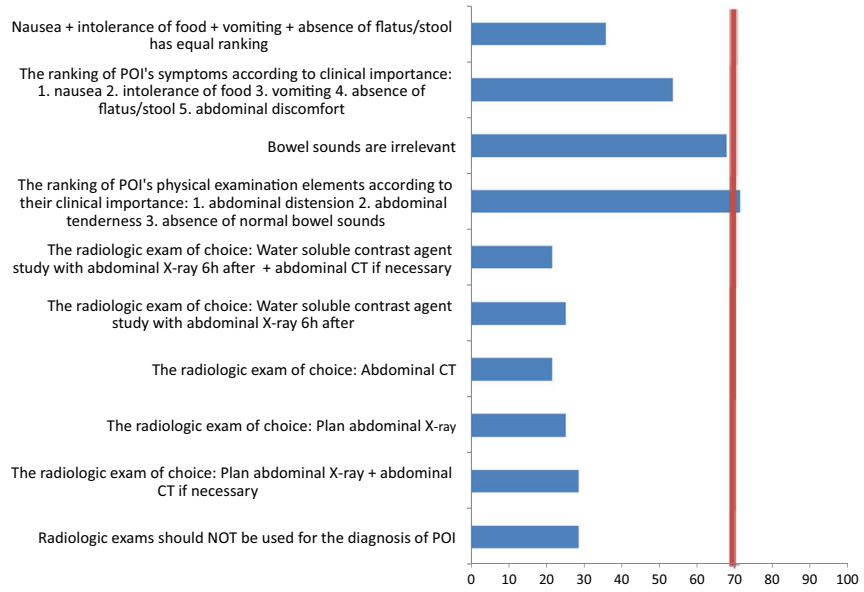

ileus and postoperative nausea and vomiting. d Indications for nasogastric tube placement and removal. e Prevention of postoperative ileus. $\mathbf{f}$ Treatment of postoperative ileus 
C

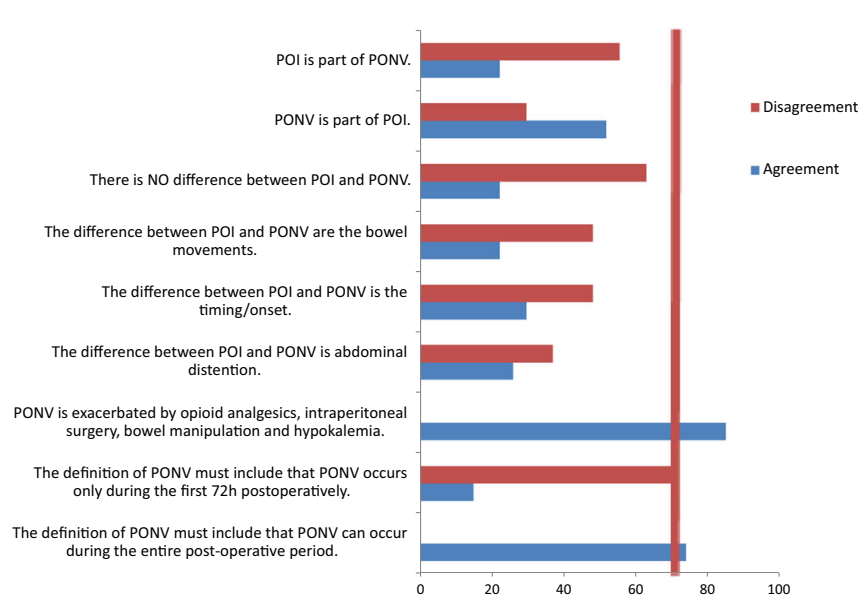

Fig. 2 (continued)

From the 4th day they advocate to use "prolonged postoperative ileus" with the following specifics: nausea/vomiting, inability to tolerate an oral diet over last $24 \mathrm{~h}$, absence of flatus over last $24 \mathrm{~h}$, abdominal distension and radiologic confirmation.

\section{Choice of imaging modality in the diagnosis of POI}

Experts failed to reach consensus on the radiologic exam of choice and also on its necessity at all.

Abdominal X-ray, abdominal computed tomography (CT), gastrointestinal contrast studies, the combination of these and no imaging were all favored by some experts. According to the literature, any of these approaches is defendable [19]. A systematic review of 11 articles on POI found no single clinical manifestation to have a significant correlation with the decision to obtain X-ray films [20]. It states that abdominal $\mathrm{CT}$ is the most accurate modality to distinguish mechanical

e

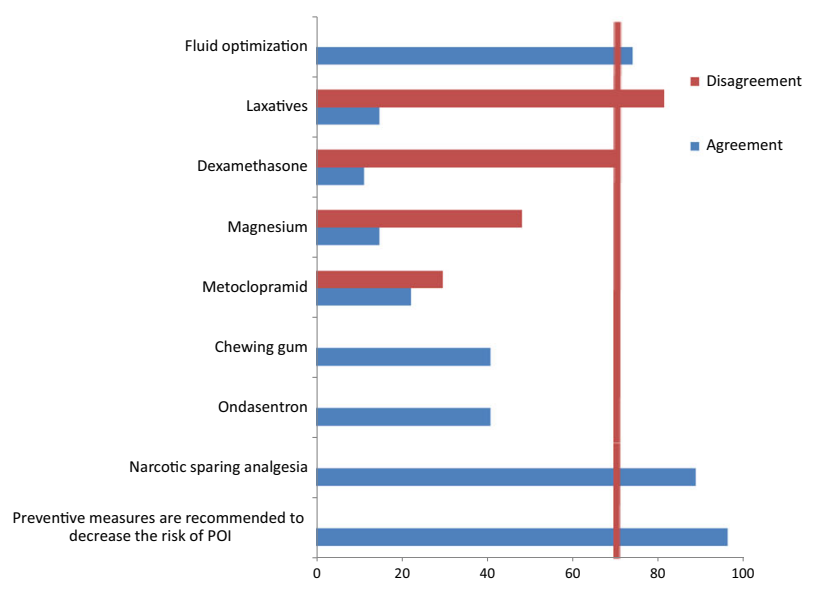

Fig. 2 (continued) d

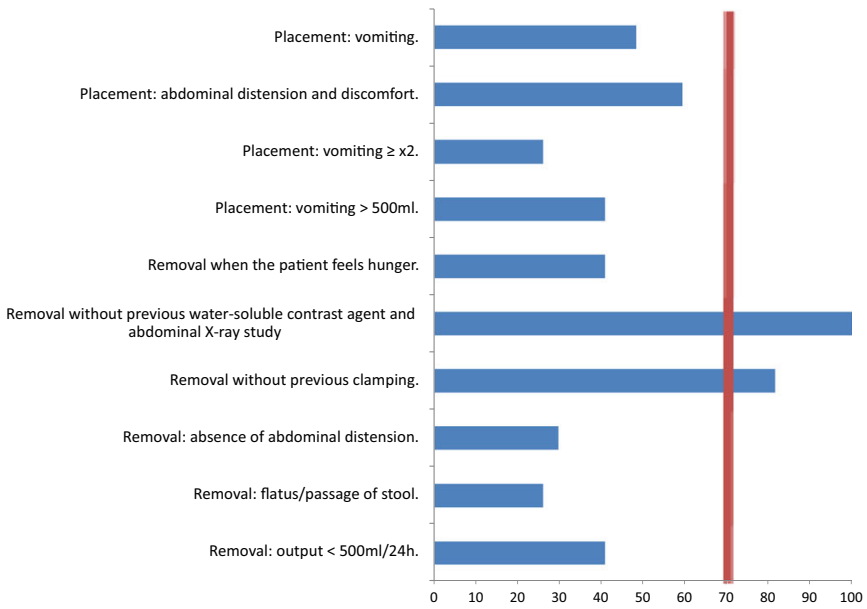

bowel obstruction from POI, and water soluble-contrast agent study is reliable in the diagnosis of POI when clinical signs and abdominal X-ray are inconclusive.

\section{Definition of postoperative vomiting and nausea}

Experts did not reach consensus on the difference between $P O I$ and PONV.

The most often mentioned differentiating items were the onset, the abdominal distention, and the bowel movements. Some suggested that these two entities were the same; some said that POI was part of PONV or vice-versa. Based on the Society for Ambulatory Anesthesia 2014 consensus guidelines [21], PONV is not limited to the first $24 \mathrm{~h}$ after surgery. Its etiology is multi-factorial, including patient-related factors (female gender; younger age; nonsmoker; history of PONV) and environmental factors (postoperative opioids; general anesthesia; emetogenic

\section{f}

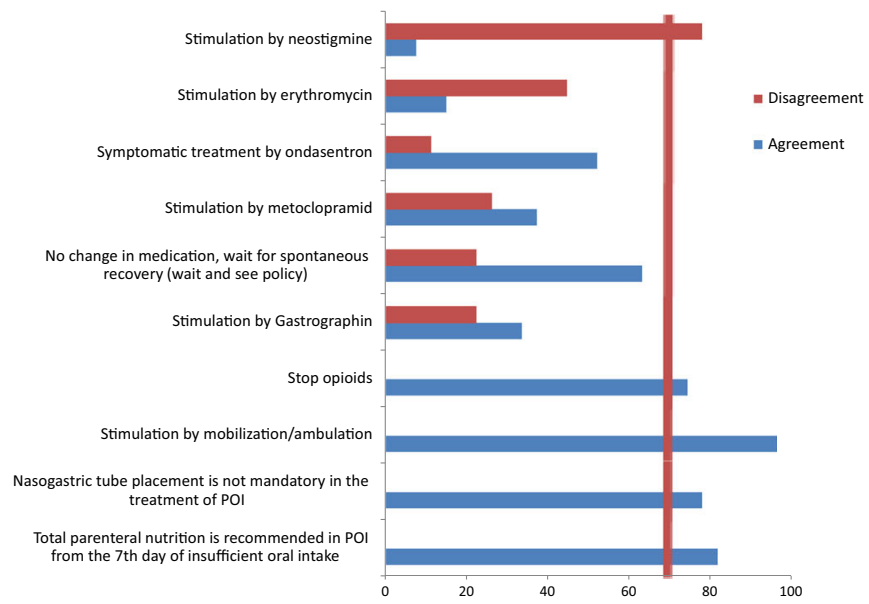


Table 2 Consensus on the definition, diagnosis, prevention, and treatment of postoperative ileus

\begin{tabular}{|c|c|c|}
\hline & & $\begin{array}{l}\text { Agreement } \\
(\%)\end{array}$ \\
\hline \multirow[t]{3}{*}{ The definition of POI } & $\begin{array}{l}\text { - Temporary inhibition of gastrointestinal motility after a } \\
\text { surgical intervention }\end{array}$ & 86 \\
\hline & - Due to non-mechanical causes & 89 \\
\hline & - Prevents sufficient oral intake & 96 \\
\hline \multirow[t]{3}{*}{ The definition of PONV } & - Can occur during the entire postoperative period & 74 \\
\hline & $\begin{array}{l}\text { - Exacerbated by opioid analgesics, intraperitoneal surgery, } \\
\text { bowel manipulation and hypokalemia }\end{array}$ & 85 \\
\hline & - Differentiation of PONV from POI is controversial & $-^{\mathrm{a}}$ \\
\hline \multirow[t]{2}{*}{ Diagnosis of POI } & $\begin{array}{l}\text { - Most relevant clinical signs: (1) abdominal distension, (2) } \\
\text { abdominal tenderness, (3) absence of normal bowel } \\
\text { sounds }\end{array}$ & 71 \\
\hline & $\begin{array}{l}\text { - No consensus on the imaging modality of choice, neither } \\
\text { on its necessity }\end{array}$ & $-{ }^{\mathrm{a}}$ \\
\hline \multirow[t]{3}{*}{$\begin{array}{l}\text { Indications of nasogastric tube } \\
\text { placement and removal in POI }\end{array}$} & $\begin{array}{l}\text { - Abdominal distension/discomfort and vomiting (no } \\
\text { consensus on the main criterion) }\end{array}$ & $60 / 48$ \\
\hline & - NG tube can be removed without previous clamping or & 81 \\
\hline & previous gastrointestinal contrast study & 100 \\
\hline \multirow[t]{4}{*}{ Prevention of POI } & $\begin{array}{l}\text { - Preventive measures are recommended to decrease the risk } \\
\text { of POI }\end{array}$ & 96 \\
\hline & - Narcotic sparing analgesia & 89 \\
\hline & - Fluid optimization & 74 \\
\hline & $\begin{array}{l}\text { - Dexamethasone and laxatives should not be used for POI } \\
\text { prevention }\end{array}$ & $70 / 81$ \\
\hline \multirow[t]{5}{*}{ Treatment of POI } & - Stimulation of ambulation/mobilization & 96 \\
\hline & - Stop of opioids & 74 \\
\hline & - Nasogastric tube placement is not mandatory & 78 \\
\hline & - Neostigmine should not be used & 78 \\
\hline & $\begin{array}{l}\text { - Total parenteral nutrition is recommended in POI from the } \\
\text { 7th day without sufficient oral intake }\end{array}$ & 81 \\
\hline
\end{tabular}

$P O I$ postoperative ileus, $N G$ nasogastric, $P O N V$ postoperative nausea and vomiting

${ }^{a}$ No consensus surgery: cholecystectomy, laparoscopy, gynecologic procedures). In this study, experts agreed that PONV can occur at any time during the postoperative period and is exacerbated by opioid analgesics, intraperitoneal surgery, bowel manipulation and hypokalemia. Gentle tissue handling by laparoscopic surgery is a valid preventive measure for POI, but has not been shown effective in PONV $[22,23]$. Hypochloremic, hypokalemic, metabolic alkalosis on presentation was proved to correlate to the number of episodes of postoperative emesis only in infants diagnosed with pyloric stenosis [24]. Its significance in the general surgical population needs further analysis.

\section{Prevention of POI}

Consensus was obtained on the use of narcotic sparing analgesia and fluid optimization. Experts also agreed that dexamethasone and laxatives should not be used for the prevention of POI.
Experts agreed that preventive measures are necessary to decrease the risk of POI. Some experts suggested the use of ondasentron, metoclopramid, chewing gum and magnesium. Concerning the pathophysiology, an inflammatory response to surgical trauma is considered as the main trigger of POI - its severity correlated in several studies with the level of circulating cytokines [20]. Immunologic response to surgery deteriorates the barrier function of the gut, allowing bacterial dislocation, stimulates immunocytes to decompose the extra-cellular matrix and enhances inflammation via the activation of mechanosensitive ion channels in the bowel wall [25]. Opioids can cause nausea and POI by modulating the transmission of peripheral and central nervous systems, leading to the inhibition of gastric emptying and to nonpropulsive contraction of the GI smooth muscles, predominantly via the $\mu$-receptors [3]. The experts' opinion was in concordance with the recent guidelines and systematic reviews: the use of non-steroidal anti-inflammatory drugs and narcotic-sparing analgesia (for example, epidural 
Table 3 List of experts

\begin{tabular}{|c|c|c|}
\hline Name & Affiliation & Region \\
\hline Nancy Baxter & Department of Surgery at St. Michael's Hospital, Toronto, Canada & America \\
\hline George J. Chang & The University of Texas MD Anderson Cancer Center, Houston, Texas, USA & America \\
\hline Conor Delaney & Department of Surgery, University Hospitals Case Medical Center, OH, USA & America \\
\hline James Fleshman & Dept. of Surgery, Washington University Medical School in St. Louis, Missouri, USA & America \\
\hline Andreas M. Kaiser & Division of Colorectal Surgery, University of South Carolina, South Carolina, USA & America \\
\hline Hermann P. Kessler & Colorectal Surgery, Cleveland Clinic, Florida, USA & America \\
\hline David W. Larson & Colon and Rectal Surgery, Mayo Clinic, Rochester, MN, USA & America \\
\hline Rodrigo O. Perez & Colorectal Surgery, University of São Paulo, Sao Paulo, Brazil & America \\
\hline Anthony J. Senagore & General Surgery, Central Michigan University, Michigan, USA & America \\
\hline Eric Weiss & Colorectal Surgery, Cleveland Clinic, Florida, USA & America \\
\hline Faris Al Aswad & General Surgery, NMC Specialty Hospital, Dubai, UAE & Asia/Oceania \\
\hline Ian Bisset & Colorectal Surgery, University of Auckland, New Zealand & Asia/Oceania \\
\hline Peter WG. Carne & Colorectal Surgery, Monash University, Victoria, Australia & Asia/Oceania \\
\hline Ho-Kyung Chun & Dept. of Surgery, Sungkyunkwan University, Seoul, South Korea & Asia/Oceania \\
\hline Sayandev Dasgupta & Dept. of Surgery, Woodlands Medical Centre, Kolkata, India & Asia/Oceania \\
\hline Alexander Engel & Academic Surgery, University of Sydney, Australia & Asia/Oceania \\
\hline Andrew G. Hill & Colorectal Surgery, University of Auckland, New Zealand & Asia/Oceania \\
\hline Hannan Jafrul & Pediatric Surgery,South Point Hospital, Chittagong, Bangladesh & Asia/Oceania \\
\hline Wai-Lun Law & Division of Colorectal Surgery, The University of Hong Kong & Asia/Oceania \\
\hline Sanjay Marwah & Dept. of Surgery, Postgraduate Inst. of Medical Sciences, Rohtak, India & Asia/Oceania \\
\hline Wilhelm Bemelman & Dept. of Surgery, University of Amsterdam, The Netherlands & Europe \\
\hline Paolo Delrio & Dept. of Surgical Science, University of Parma, Italy & Europe \\
\hline Daniel Dindo & Dept. of Surgery, Stadtspital Triemli, Zürich, Switzerland & Europe \\
\hline Eloy Espin & Dept. of Surgery, Universidat Autónoma de Barcelona, Spain & Europe \\
\hline Alois Fürst & Dept. of Surgery, Caritas-Krankenhaus St. Josef, Regensburg, D & Europe \\
\hline Ethem I. Gecim & Dept. of Surgery, Ankara University School of Medicine, Turkey & Europe \\
\hline Soren Laurberg & Dept. of Surgical Gastroenterology, Aarhus University, Denmark & Europe \\
\hline Olle Ljundqvist & Dept. of Surgery, Örebro University Hospital, Sweden & Europe \\
\hline Yves Pannis & Dept. of Colorectal Surgery, Beaujon Hospital, Clichy, France & Europe \\
\hline Stefan Post & Dept. of Surgery, University of Heidelberg, Mannheim, Germany & Europe \\
\hline Harald Rosen & Dept. of Surgery, Danube Hospital-SMZ-Ost, Vienna, Austria & Europe \\
\hline Emmanuel Tiret & Dept. of Surgery, Saint-Antoine Hospital, Paris, France & Europe \\
\hline Petr Tsarkov & Dept. of Colorectal and Pelvic Floor Surgery, Russian Academy of Medical Sciences, Moscow & Europe \\
\hline Arne Wibe & Dept. of Cancer Research, Norwegian University of Science and Technology, Trondheim, Norway & Europe \\
\hline Des Winter & Dept. of Surgery, St Vincent's University Hospital, Dublin, Ireland & Europe \\
\hline
\end{tabular}

analgesia for open procedures) proved to reduce the time of bowel function recovery [26, 27]. As a cardinal point of prevention, physiologic research also supports the aim for "zero fluid balance" in the perioperative phase, to avoid intestinal edema and electrolytic disturbances, and as a consequence, gut dysfunction [28]. With regards to pharmacologic agents for the prevention of POI, a Cochrane Review investigated 15 prokinetic substances and found only one (alvimopan, a selective $\mu$-receptor antagonist) with a reproducible clinical benefit [29]. Recent trials showed significantly faster bowel function recovery with magnesium and chewing gum use [30, 31].

\section{Indications of nasogastic (NG) tube placement and removal}

This study did not identify one main indication for NG tube placement in POI.

However, several acceptable indications were sustained by experts. Abdominal distension/tenderness and vomiting $>500 \mathrm{ml}$ were the most popular ones, while intractable nausea was also mentioned. Consensus was achieved on the nonnecessity of clamping test and gastrointestinal contrast series before NG tube removal. Forty percent of the experts would remove NG tube when the patient feels hunger. There are no 
published algorithms for NG tube placement and removal. Van Bree et al. performed a statistical analysis to evaluate the predictive value of different indicators in a prospective study where colonic transit was scintigraphically assessed from postoperative day 1 to 3 [32]. They concluded that tolerance of solid food and defecation, but not time to first flatus, best indicates recovery of GI transit.

\section{Treatment of POI}

The experts reached consensus on stimulation of ambulation, cessation of opioids and administration of parenteral nutrition from the 7th day without sufficient oral intake in patients with POI.

NG tube placement was not deemed mandatory; however, it is a basic measure to decompress the small bowel, to comfort the patient and to reduce the risk of bronchoaspiration. These measures are consistent with an evidence-based recommendation on the management of POI, published in 2013 [33]: ambulate regularly (Grade C); pain relief with paracetamol, non-steroidal anti-inflammatory drugs (NSAIDs); Tramadol, opiates in reserve (Grade A); parenteral nutrition after 7 days (Grade A). Other therapeutic interventions failed to obtain consensus. Of note, $63 \%$ of experts would not make any changes in the medication of patients presenting POI and would wait spontaneous recovery. Water-soluble contrast agents can be used as diagnostic tool and therapeutic attempt at the same [34], but only one third of experts supported their utility. Ondasentron, erythromycin and metoclopramid were mentioned by some experts, without achieving consensuson the other hand, these products have never been proved to foster POI recovery [3]. Neostigmine is a parasympathomimetic drug that acts as a reversible acetylcholinesterase inhibitor. Some studies have shown modest effectiveness in treating acute colonic pseudo-obstruction, but the clinical usefulness of neostigmine in postoperative patients may be limited by adverse effects including abdominal cramps, excess salivation, vomiting, and bradycardia [35]. Experts in this study agreed that neostigmine is not to be used to alleviate POI. In the literature, various clinical end-points of POI have been used, making difficult to compare the efficacy of therapeutic interventions.

\section{Strengths and limitations}

The study has some limitations. First, we set the level of agreement for consensus at only $70 \%$. However, there is great variability among Delphi studies on the rule to determine consensus. Setting a percentage level of agreement between 51 and $80 \%$ before the commencement of the study is an accepted practice [36, 37]. Secondly, we invited 35 colorectal surgeons as experts and therefore might have missed view-points of non-colorectal surgeons and/or anesthesiologists who are also involved in the management of PONV and POI. The number of experts that represents an adequate sample in a Delphi study remains unknown. The quality of expert panel, rather than its size guarantees probably better representativeness. 35 experts from 5 continents provided a variety of opinions with international relevance. We realize, however, that the selection of the experts who were initially invited to participate $(n=61)$ was somewhat arbitrary and might be a source of bias. The main strengths of this study were diversity of nationalities in the panel, truly representing a world experience. The experts were active and engaged with the Delphi, reflecting their interest in the topic. The possibility of leaving comments at the end of each sub-topic was appreciated and used by experts. The comments to the 2 nd round were included in the 3rd questions and happened to be subjected to consensus. The use of online interface was user-friendly and allowed real-time communication.

\section{Implications and future research}

This Delphi study confirms that recent evidence-based recommendations for the treatment of POI are widely supported by international experts. The consensual items of the study may guide decision-making in daily practice whereas the identified areas of controversy may be subject of future research. The study provides a practical definition of POI, suggests preventive measures and therapeutic steps for the general surgical population, including precise indication of parenteral nutrition or NG tube placement/removal. The concept of multimodal rehabilitation after surgery aims to shorten the postoperative recovery and hospital stay and to cut complication-related costs [38]. POI and PONV are among the most frequent complications following abdominal surgery: they prevent oral intake and ambulation and delay discharge.

\section{Conclusion}

This consensus research established a definition for POI and adopted measures to prevent, diagnose, and treat it. Accordingly, POI occurs temporarily after a surgical intervention due to non-mechanical causes and prevents sufficient oral intake. Prophylaxis should include narcotic sparing analgesia and fluid optimization; whereas treatment should rely on stimulation of ambulation and opioid withdrawal. The consensual definition may support further studies on POI by improving homogeneity among them and thus improving the quality of research in this field. The preventive and therapeutic measures obtained in this Delphi may guide clinicians in the establishment of multimodal caremaps and in the choice of therapeutic options for patients presenting with POI. This study may contribute to future observational cohort studies to validate these consensual findings. Areas of remaining controversy consist 
of the difference of PONV and POI and the imaging modality of choice for POI (if necessary at all).

Acknowledgments We thank the participating experts listed in Table 3 for their time, commitment, and valuable input to this study.

Author contributions Study conception and design: D Hahnloser, O Gié, M Hubner, D Gero

Acquisition of data: D Gero, O Gié

Analysis and interpretation of data: D Gero, O Gié, M Hübner, D Hahnloser

Drafting of manuscript: D Gero, D Hahnloser

Critical revision of manuscript: O Gié, M Hübner, N Demartines, D Hahnloser

\section{Compliance with ethical standards}

Funding No external sources involved.

Conflict of interest The authors declare that they have no conflict of interest.

Ethical approval All procedures performed in studies involving human participants were in accordance with the ethical standards of the institutional research committee and with the 1964 Helsinki declaration and its later amendments or comparable ethical standards.

Informed consent Informed consent was obtained from all individual participants included in the study.

\section{References}

1. Wolthuis AM, Bislenghi G, Fieuws S, de Buck van Overstraeten A, Boeckxstaens G, D'Hoore A (2016) Incidence of prolonged postoperative ileus after colorectal surgery: a systematic review and meta-analysis. Color Dis 1:O1-O9

2. Vather R, Trivedi S, Bissett I (2013) Defining postoperative ileus: results of a systematic review and global survey. J Gastrointest Surg 5:962-972

3. Holte K, Kehlet H (2000) Postoperative ileus: a preventable event. Br J Surg 11:1480-1493

4. Asgeirsson T, El-Badawi KI, Mahmood A, Barletta J, Luchtefeld M, Senagore AJ (2010) Postoperative ileus: it costs more than you expect. J Am Coll Surg 2:228-233

5. Vather R, O'Grady G, Bissett IP, Dinning PG (2014) Postoperative ileus: mechanisms and future directions for research. Clin Exp Pharmacol Physiol 5:358-370

6. Lee SY, Park KJ, Ryoo SB, HK O, Choe EK, Heo SC (2014) Early postoperative small bowel obstruction is an independent risk factor for subsequent adhesive small bowel obstruction in patients undergoing open colectomy. World J Surg 11:3007-3014

7. Doorly MG, Senagore AJ (2012) Pathogenesis and clinical and economic consequences of postoperative ileus. Surg Clin North Am 2:259-272

8. Kranke P, Thompson JP, Dalby PL, et al. (2015) Comparison of vestipitant with ondansetron for the treatment of breakthrough postoperative nausea and vomiting after failed prophylaxis with ondansetron. Br J Anaesth 3:423-429

9. Hsu CC, Sandford BA (2007) The Delphi technique: making sense of consensus. Practical Assessment, Research \& Evaluation 10:1-8

10. Fiore JF Jr, Bialocerkowski A, Browning L, Faragher IG, Denehy L (2012) Criteria to determine readiness for hospital discharge following colorectal surgery: an international consensus using the Delphi technique. Dis Colon rectum 4:416-423

11. Murphy MK, Black NA, Lamping DL, et al. (1998) Consensus development methods and their use in clinical guideline development. Health Technol Assessment 2:1-88

12. Jones J, Hunter D (1995) Consensus methods for medical and health services research. BMJ 311:376-380

13. Hasson F, Keeney S, McKenna H (2000) Research guidelines for the Delphi survey technique. J Adv Nurs 32:1008-1015

14. Roberts DM, Yates C, Megarbane B, et al. (2015) Recommendations for the role of extracorporeal treatments in the management of acute methanol poisoning: a systematic review and consensus statement. Crit Care Med 2:461-472

15. Swamy M, Venkatachalam S, McLachlan J (2014) A Delphi consensus study to identify current clinically most valuable orthopaedic anatomy components for teaching medical students. BMC Med Educ 14:230

16. Dijkstra FA, Bosker RJ, Veeger NJ, van Det MJ, Pierie JP (2015) Procedural key steps in laparoscopic colorectal surgery, consensus through Delphi methodology. Surg Endosc 9:2620-2627

17. Jacobs M, Henselmans I, Macefield RC, et al. (2014) Delphi survey to identify topics to be addressed at the initial follow-up consultation after oesophageal cancer surgery. Br J Surg 13:1692-1701

18. Bonrath EM, Grantcharov TP (2015) Contemporary management of paraesophaegeal hernias: establishing a European expert consensus. Surg Endosc 8:2180-2195

19. Sandrasegaran K, Maglinte DD (2015) Imaging of small bowelrelated complications following major abdominal surgery. Eur $\mathrm{J}$ Radiol 3:374-386

20. Wu Z, Boersema GS, Dereci A, Menon AG, Jeekel J, Lange JF. (2014) Clinical endpoint, early detection, and differential diagnosis of postoperative ileus: a systematic review of the literature. Eur Surg Res, 3-4:127-138.

21. Gan TJ, Diemunsch P, Habib AS, et al. (2014) Consensus guidelines for the management of postoperative nausea and vomiting. Anesth Analg 1:85-113

22. Shussman N, Brown MR, Johnson MC, Da Silva G, Wexner SD, Weiss EG (2013) Does nasogastric tube decompression get used less often with laparoscopic and hand-assisted compared with open colectomy? Surg Endosc 12:4564-4568

23. Sirivanasandha $P$ (1995) Postoperative nausea vomiting (PONV): influence of bowel manipulation during intraabdominal surgery. J Med Assoc Thail 10:547-553

24. St Peter SD, Tsao K, Sharp SW, Holcomb GW, Ostlie DJ (2008) Predictors of emesis and time to goal intake after pyloromyotomy: analysis from aprospective trial. J Pediatr Surg 11:2038-2041

25. Wehner S, Vilz TO, Stoffels B, Kalff JC (2012) Immune mediators of postoperative ileus. Langenbeck's Arch Surg 4:591-601

26. Gustafsson UO, Scott MJ, Schwenk W, et al. (2013) Guidelines for perioperative care in elective colonic surgery: enhanced recovery after surgery (ERAS((R))) society recommendations. World J Surg, 2:259-284.

27. Pöpping DM, Elia N, Van Aken HK, et al. (2014) Impact of epidural analgesia on mortality and morbidity after surgery: systematic review and meta-analysis of randomized controlled trials. Ann Surg 6:1056-1067

28. Lobo DN (2004) Fluid, electrolytes and nutrition: physiological and clinical aspects. The Proceedings of the Nutrition Society 3:453-466

29. Traut U, Brügger L, Kunz R, et al. (2008) Systemic prokinetic pharmacologic treatment for postoperative adynamic ileus followingabdominal surgery in adults. Cochrane Database Syst Rev 1:CD004930

30. Shariat Moharari R, Motalebi M, Najafi A, et al. (2013) Magnesium can decrease postoperative physiological ileus and postoperative pain in major non laparoscopic gastrointestinal surgeries: a randomized controlled trial. Anesth Pain Med 1:e12750 
31. van den Heijkant TC, Costes LM, van der Lee DG, et al. (2015) Randomized clinical trial of the effect of gum chewing on postoperative ileus and inflammation in colorectal surgery. Br J Surg 3:202-211

32. van Bree SH, Bemelman WA, Hollmann MW, et al. (2014) Identification of clinical outcome measures for recovery of gastrointestinal motility in postoperativeileus. Ann Surg 4:708-714

33. Vather R, Bissett I (2013) Management of prolonged post-operative ileus: evidence-based recommendations. ANZ J Surg 5:319-324

34. Branco BC, Barmparas G, Schnuriger B, Inaba K, Chan LS, Demetriades D (2010) Systematic review and meta-analysis of the diagnostic and therapeutic role of water-soluble contrast agent in adhesive small bowel obstruction. Br J Surg 4:470-478
35. Zeinali F, Stulberg JJ, Delaney CP (2009) Pharmacological management of postoperative ileus. Can J Surg 2:153-157

36. Elledge RO, McAleer S (2015) Planning the content of a brief educational course in maxillofacial emergencies for staff in accident and emergency departments: a modified Delphi study. Br J Oral Maxillofac Surg 2:109-113

37. Nagpal K, Arora S, Abboudi M, et al. (2010) Postoperative handover: problems, pitfalls, and prevention of error. Ann Surg 1:171-176

38. Fesharakizadeh M, Taheri D, Dolatkhah S, Wexner SD (2013) Postoperative ileus in colorectal surgery: is there any difference between laparoscopic and open surgery? Gastroenterol Rep (Oxf) 2:138-143 